\title{
Research on the Quantitative Dynamic Structure of Shocks in
}

\section{Macroeconomics Business Cycle}

\author{
Liangxin Li \\ Department of Finance, Hunan International Economics University \\ Changsha 410205, Hunan, China \\ E-mail: apolo1@sina.com
}

\begin{abstract}
In this paper, we attempt to study quantitatively the effects on macroeconomics due to different shocks. The interactions between different shocks are the major subjects. Furthermore, the dynamics of shocks, i.e, the time evolutions of shocks, and the propagation of shocks are studied in detail by using the quantitative models. The approach of this paper can be applied to any economic shocks, particularly, price shocks, technology shocks, unemployment shocks, and outer capital flow shocks. As an example, we investigate the interaction of price shock and exchange rate shock. At the same time, we also discuss the macroeconomic policy's effects on economic business cycle.
\end{abstract}

Keywords: Shock, Macro economy, Propagation, Coupling, Economic policy, Business cycle

\section{Introduction}

As well known, the economy fluctuates as a whole in the short run. What is the best way to explain short run fluctuations in output, employment, exchange rates, inflation rates, and price level? How should monetary and fiscal policy respond to these fluctuations to achieve the best economic performance? These are the central questions of modern macroeconomics. Unfortunately, there are no clear ideas on the answers so far. As a matter of logic, the output of the economy can fluctuate for two reasons: either because the natural rate of output fluctuates or because the output of the economy has deviated from its natural rate, i.e., the shocks. Here, we focus on the shocks' quantitative behavior, especially, the combination effects of shocks as in the real case. Historically, in exploring these business cycle questions, people have focused on five related activities: empirically identifying the effects of exogenous shocks to the business cycle, with an emphasis on shocks to monetary and fiscal policy (Christinao,etal,1998); assessing the empirical plausibility of alternative structural business cycle models (Barro, 1995); constructing new general equilibrium business cycle model (Obstfeld,1995); exploring the nature of actual and optimal monetary policy, and analyzing the impact of different institutions within which policy is conducted(Bernanke,etal,1998); and considering the role of monetary, fiscal, and regulatory policy as well as self-fulfilling expectations in causing recent currency crises ( Bernanke, etal, 1997, pp91-142 ).

No shock-coupling models have been built up so far (Boshen, etal, 1998, pp91-200; Bernanke, 1986, pp49-99; Campbell, 1998, pp102-115; Razin, etal, 2002; Christiano, etal, 1992, pp335-370). But in reality, the economy often experiences many combinations of fluctuations around the expected values, such as the high inflation rates often accompanied by low unemployment, as in 1960s of US economy; high oil price usually can relate itself to low GDP growth as in 1970s of the world economy; low money supply generally can find the high unemployment and low inflation with itself, as in 1980s' US economy. These examples clearly show the coupling effects between different shocks. The economy is so complex that it is not possible to identify which effects are caused by which shocks (Liang-Xin Li, 2008, pp81-87). What we can see in realistic economy is the coupling effects of all shocks.

To advance along this trend, this paper makes a brave attempt to tackle the complex shocks dynamics in a straight quantitative style. In the second section, we are going to present our theoretical models, different results of the shocks are also derived; Section 3 is trying to discuss different economic policy regime's influence over the shocks' effects and make a real contact with the economy. And two extensions are made with (1) multi-shock models and (2) time evolution of shocks. In section 4, we summarize our results.

\section{The model}

Our starting point is the loss function, which is given in the following in our model: 


$$
L=\left(y-y^{*}\right)^{2}+b_{1} \delta_{1}^{2}+b_{2} \delta_{2}^{2}+\alpha_{1} u_{1}^{2}+\alpha_{2} u_{2}^{2}+C\left(\delta_{1,} \delta_{2}\right)
$$

and

$$
y=\bar{y}+a_{1}\left(\delta_{1}-\delta_{1}^{E}\right)+a_{2}\left(\delta_{2}-\delta_{2}^{E}\right)+c_{1} u_{1} u_{2}-u_{1}-u_{2}
$$

as the generalized Phillips curve. Where $u_{1}$ is the exchange rate shock, for example, $u_{2}$ is the price shock. $c_{1}$ the coupling constant between the two shocks. And $\delta_{1}, \delta_{2}$ are the changes of exchange rate level and the price level respectively, i.e., $\delta_{1} \equiv U-U_{-1}, \delta_{2}=P-P_{-1}$ with the past years exchange rate level and price level as the adapted expectations for the corresponding levels of the current year. $\mathrm{C}$ is the extra cost function of the economy for the particular deltas. $b_{1}, b_{2}, \alpha_{1}, \alpha_{2}, a_{1}, a_{2}$ are the constants to describe the contributions of price and exchange rate's contribution to the corresponding functions.

To derive the results needed, we minimize the loss function in Eq.1 The minimized loss function value for flexible policy regimes on deltas is:

$$
L^{\text {FLEX }}=\frac{4 b_{1} b_{2}\left(a_{1}^{2} b_{2}+b_{1}^{3}+b_{1} b_{2}\right)}{\left(a_{1}^{2} b_{2}+a_{2} b_{1}^{2}+b_{1} b_{2}\right)^{2}} A^{2}
$$

and for fixed deltas regime, the loss function value is:

$$
L^{F I X}=A^{2}
$$

Suppose for any given deltas, only when delta are either so low that $L^{F L E X}+\bar{c}<L^{F I X}$ or so high that $L^{F L E X}+\underline{c}<L^{F I X}$, the deltas' policy will be in effective as in the equations of deltas. To solve these two boundaries, we treat $\delta_{2}, u_{2}$ as constants for any particular moments and solve for $u_{1}$ 's boundary (we will get the full picture of $u_{1}, u_{2}$ in a plane drawing later.).

\section{Results and Discussions.}

We first explore the results of the model, especially investigate the coupling effects between the price shock and the exchange rate shock.

\section{1) The results of the model:}

In Figure 1, we show the fixed points: $E \delta_{1}=\delta_{1}^{E}$.

\section{Insert Figure 1 here.}

One can see that different coupling strength, i.e., different interaction strength for different economy will shift the fixed points into different positions. We can use this effect to get the desired fixed points by performing suitable economic policy. The coupling of the shocks of price level and the exchange rates can be seen in the Asia financial crisis: the devaluation of the currency leads to the exchanged collapsed, i.e., the fix points shifted remarkably due to the price shocks.

The results in Fig. 1 shows if we change the coupling constants, the fixed points of price level can be shifted above or below. In reality, we can propagate an exchange rates shock into the economy to reduce or enlarge the inflation level. It might be a demonstration of the Argentina economy: the flexible exchange rate policy leads to the devaluation of the Argentina money.

In Figure 2, we will draw the boundary for $\delta_{1}, \delta_{2}$ in an equal footing which shows that inside the boundary, the minimization doesn't have any effects on the deltas due to the cost to implement the policy is bigger than not to do anything; outside the region, the policy for deltas will get into effect because of the huge cost if we don't control the parameter levels.

\section{Insert Figure 2 here.}

One can build up the warning system for the economy by using this model to steer the economy in all time as needed.

\section{2) Two extensions to the model:}

To get closer to the real economy, we extend our models into two different directions: 1) multi-shocks regime; 2) the time evolution of shocks in a coupled system. 


\section{(i) Extensions to multi-shocks:}

In general, our model can be extended to multi-shocks coupling. In this case, the loss function can be written as:

$$
\begin{aligned}
& L=\left(y-y^{*}\right)^{2}+\sum_{j=1}^{N} b_{j} \delta_{j}^{2}+\sum_{j=1}^{N} \alpha_{j} u_{j}^{2}, \\
& y=\bar{y}+\sum_{j=1}^{N} a_{j}\left(\delta_{j}-\delta_{j}^{E}\right)+\sum_{j=1}^{N} \kappa_{j} u_{j}+\sum_{j>k=1}^{N} c_{j k} u_{j} u_{k}+O(\text { uuu })
\end{aligned}
$$

Starting from this model, the general quantitative results frame can be drawn from the model.

\section{(ii) Extensions to time propagation:}

To incorporate the time propagation of shocks, we need to extend the loss function to include the Shock's speed term, i.e., the kinetic energy term as shown in the following:

$$
\begin{aligned}
& L=\left(y-y^{*}\right)^{2}+\sum_{j=1}^{N} \varepsilon_{j}\left(u_{j}^{\prime}\right)^{2}+\sum_{j=1}^{N} b_{j} \delta_{j}^{2}+\sum_{j=1}^{N} \alpha_{j} u_{j}^{2}, \\
& \text { where }, \ldots . u_{j}^{\prime}=\frac{\partial u_{j}}{\partial t} . \\
& y=\bar{y}+\sum_{j=1}^{N} a_{j}\left(\delta_{j}-\delta_{j}^{E}\right)+\sum_{j=1}^{N} \kappa_{j} u_{j}+\sum_{j>k=1}^{N} c_{j k} u_{j} u_{k}+O(\text { uuu })
\end{aligned}
$$

To get the minimized loss function, we will present the time propagation dynamics of the shocks. Again, the constants are the reflection response of the different economy system. Due to the nonlinear coupling, the chaotic behavior could be realized in some economy systems.

We treat the loss function as a dynamic Hamilton system. By minimizing the loss function, we first get the dynamic equations for the two-shock system:

$$
\begin{aligned}
& \varepsilon_{1} u_{1}^{\prime \prime}=-\alpha_{1} u_{1}-2\left(\bar{y}-y^{*}+\sum_{j=1}^{2} a_{j}\left(\delta_{j}-\delta_{j}^{E}\right)+\sum_{j=1}^{2} \kappa_{j} u_{j}+c_{1} u_{1} u_{2}\right)\left(1+c_{1} u_{2}\right), \\
& \varepsilon_{2} u_{2}^{\prime \prime}=-\alpha_{2} u_{2}-2\left(\bar{y}-y^{*}+\sum_{j=1}^{2} a_{j}\left(\delta_{j}-\delta_{j}^{E}\right)+\sum_{j=1}^{2} \kappa_{j} u_{j}+c_{1} u_{1} u_{2}\right)\left(1+c_{1} u_{1}\right) .
\end{aligned}
$$

The solution for Equation.7 is very rich in structure due to different constants in the equation. Generally, one can obtain a coupled oscillation of two shocks:

$$
\begin{aligned}
& u_{1}=u_{1}^{0}+U_{1} \sin \left(\omega_{i} t\right), \\
& u_{2}=u_{2}^{0}+U_{2} \sin \left(\omega_{i} t\right), \\
& \text { where }, i=1,2
\end{aligned}
$$

This is a purely periodic solution for the shocks as shown if the parameter is such that $\omega$ are purely real as shown in Fig.3.

\section{Insert Figure 3 here.}

For further discussion, let us take a look at the solution for $\omega$ as a function of the coupling constants:

$$
\omega_{1,2}^{2}=\frac{2 \kappa_{1}+2 \kappa_{2}+a_{1}+\alpha_{2} \pm \sqrt{\left(a_{1}+2 \kappa_{1}-a_{2}-2 \kappa_{2}\right)^{2}+16 \kappa_{1} \kappa_{2}}}{2}
$$

\section{Case 1:}

for the $\omega$ purely real, This case will give us a purely oscillation pattern of shocks as shown in Figure 4 above.

\section{Case 2:}

$\omega \quad$ is purely imaginary: we will get either blow-up into a economy crisis or booming or a decay shock as usual.

\section{Case 3:}

The complex $\omega$.For this situation, we will get shocks which evolve as decayed or blow-up oscillations.

In the following diagram [Fig.4], a whole picture in the coupling constants plane is drawn for all cases discussed. 


\section{Insert Figure 4 here.}

We can put a friction term into Equation. 7 to reflect the information delay, limitation and asymmetry or the government's inefficiency, i.e., the bias of the economy from the strong free market assumptions: all economic related items are freely flow through all the elements of the economy.

$$
\begin{aligned}
& \varepsilon_{1} u_{1}^{\prime \prime}=-\sigma_{1} u_{1}^{\prime}+\left(-2 \kappa_{1}-\alpha_{1}\right) u_{1}-2 \kappa_{2} u_{2}-2 A \\
& \varepsilon_{2} u_{2}^{\prime \prime}=-\sigma_{2} u_{2}^{\prime}+\left(-2 \kappa_{2}-\alpha_{2}\right) u_{2}-2 \kappa_{1} u_{1}-2 A \\
& \text { with } \\
& A=\bar{y}-y^{*}+\sum_{j=1}^{2} a_{j}\left(\delta_{j}-\delta_{j}^{E}\right)
\end{aligned}
$$

Where $\sigma$ are the effective friction constants for a particular economy. This will always contribute a decay term to the solutions besides anything else. Whether the combined final results are decaying or blowing up depends on the competition between $\sigma$ and $\alpha$.

Also, these nonlinear effects could lead to chaotic behaviors for some coupling constants, i.e., the economic states could bifurcate in one points due to small perturbations

\section{Summary}

In summary, we have developed a model to quantify the effects of shocks, especially, the shocks' coupling effects on the economy. From our model, we found that: 1) shocks will make the economy fluctuate, 2) different economic policy will direct the shocks to different pattern and influence the economy's different aspects. 3) for our example, we found that the price shocks' coupling with exchange rates will shift the fixed exchange rates points and will be different for different coupling strength. 4) we build up the effective time propagation model which involves the nonlinear interactions of multi-shocks. 5) effective (Note 1) coupling strength can be drawn from the quantitative consideration of the data.

For the time evolution related issues, the major concern is that the speed terms in the loss function. How quantitatively measure the cost to the government due to the remarkable change of the shock regime in the economy is the key for us to establish the approach here. Of course, the historic data is always a pool to draw some the concrete results.

\section{Acknowledgements}

The author would like to thank Dr. Tian Wenzhao of Chinese Nationality Stocks Research Center for reading this manuscript.

\section{References}

A. Razin and E. Sadka. (2002). A Brazilian Debt-Crisis Model, NBER working paper.

B.Bernanke, M. Gertler and S. Gilchrist. (1998). NBER working paper 6455. The Financial Accelerator in a Quantitative Business Cycle Framework.

Barro, Robert. (1977). Unanticipated Money Growth and Unemployment in the United States. American Economic Reviews, Vol 67, No.2, Page 101-115.

Bernanke, Ben S. (1986). Alternative Explanations of the Money-Income Correlations. Carnegie-Rochester Conference Series on Public Policy, Vol. 25, pages 49-99.

Bernanke, Ben S., MaRK Gertler and Mark Watson. (1997). Systematic Monetary Policy and the Effects of Oil Price Shocks", Brookings Papers on Economic Activity, pages 91-142.

Boshen, John F., and Leonard O. Mills. The Effects of Countercyclical Monetary Policy on Money and Interest Rates: An Evaluation of Evidence from FOMC Documents. Working PAPER. 91-200, Federal Reserve Bank of Philadelphia.

Campbell, Jeff. (1998). Cyclical Job Creation, Job Destruction and Monetary Policy. Manuscript, pages 102-115, University of Rochester.

Christiano, Lawrence J. and Martin Eichenbaum. (1992). Identification and the Liquidity Effect of Monetary Policy Shock"in Politcal Economy, Growth and Business Cycles, edited by Alex Cukierman, Zvi Hercowitz, and Leonardo Leiderman, Cambridge and London: MIT Press, pages 335-370.

L.J. Christinao, M. Eichenbaum and C. L. Evans. (1998). NBER working paper 6400, “ Monetary policy shocks: 
what have we learned and to what end?".

Li Liang-Xin. (2009). A Realistic Approach to Calculate VaR. International Journal of Finance and Economics, pages 81-87.

M. Obstfeld. (1995). NBER working paper 5285, "Models of Currency Crises With Self-Fulfilling Features".

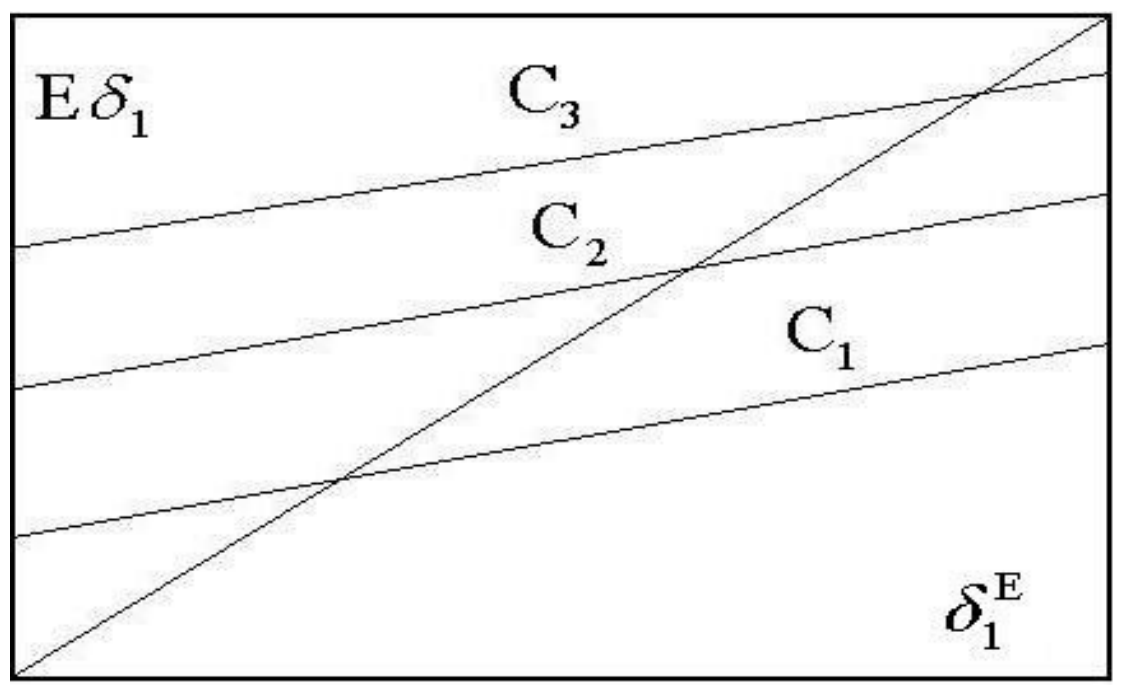

Fig. 1. The fixed points with different coupling constants: $\mathrm{c} 1<\mathrm{c} 2<\mathrm{c} 3$

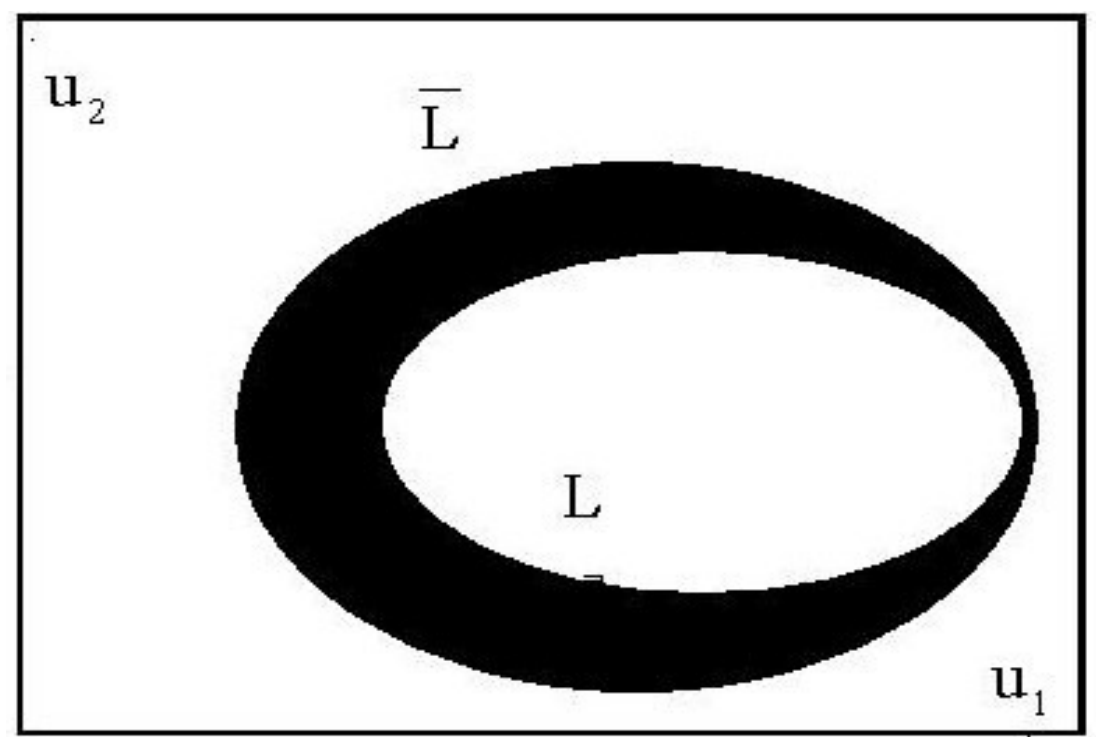

Fig. 2. The boundary for the $u_{1}, u_{2}$ to fulfill the loss functions, i.e., only in the unshaded area, the policy will be effective for $\delta_{1}, \delta_{2}$ 
u

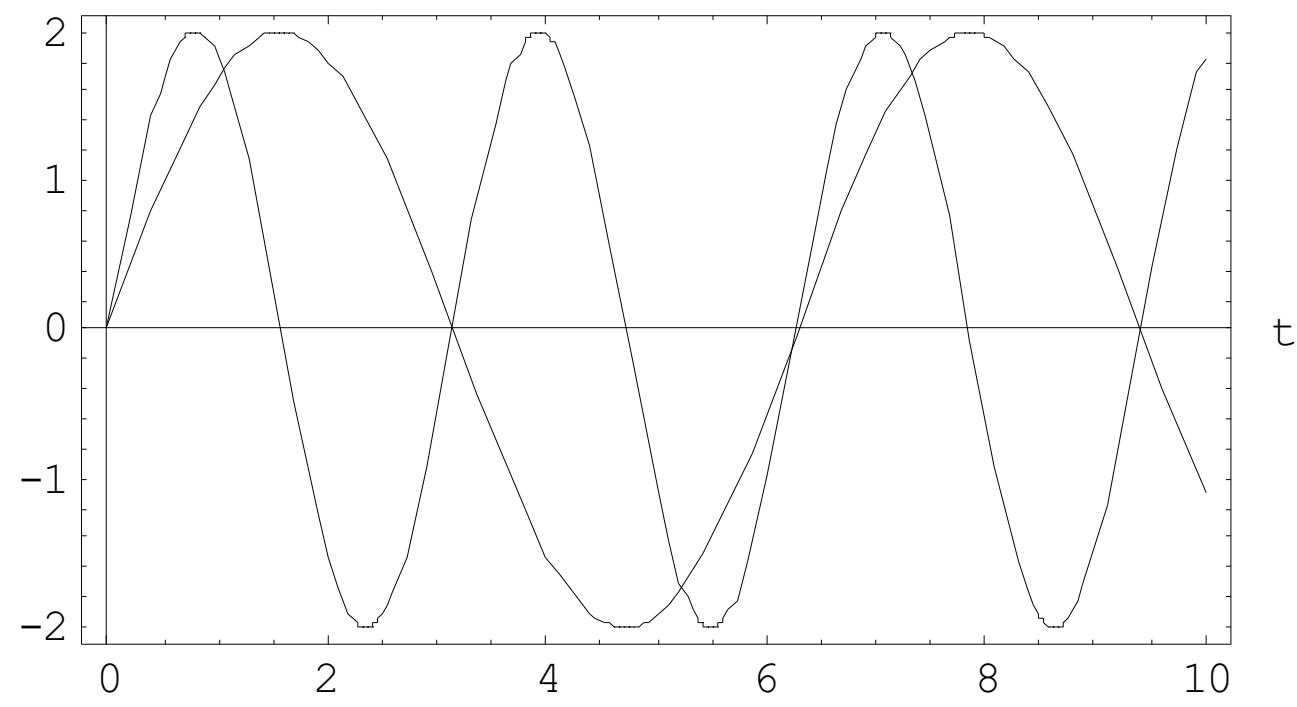

Fig. 3. The oscillation case for both shocks

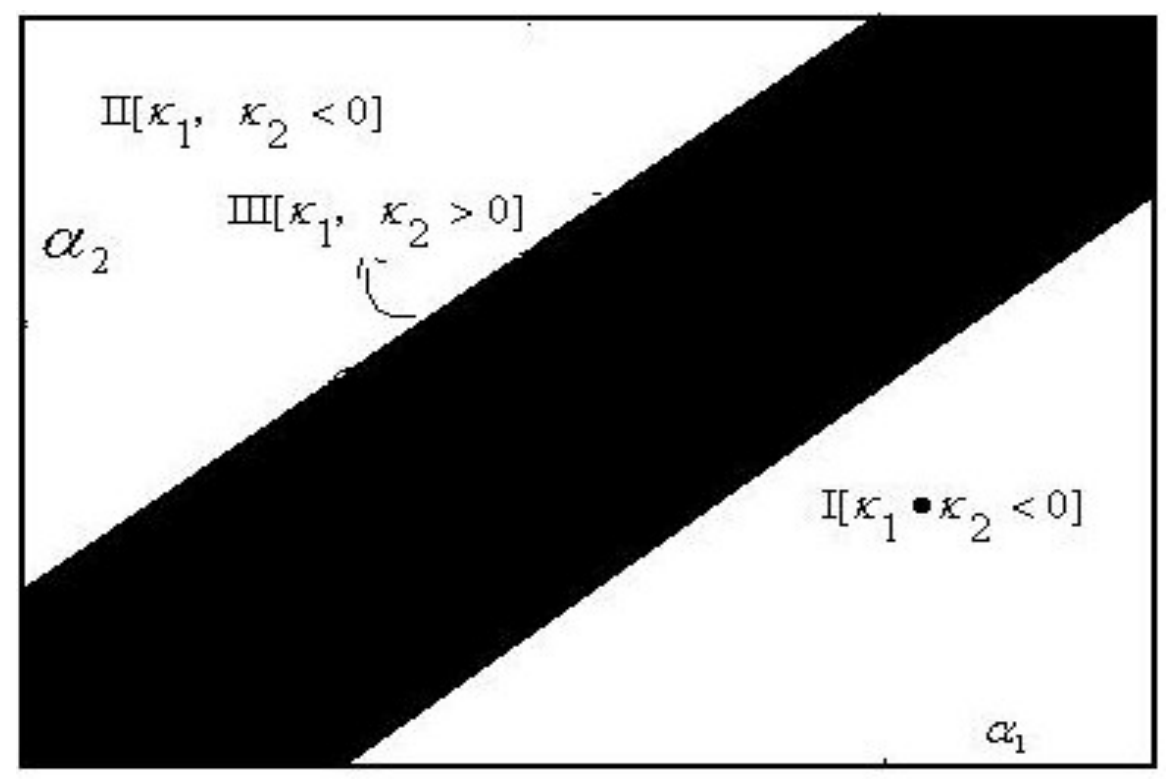

Fig. 4. Shows the 3 cases' regimes for the $\alpha \mathrm{s}$ 